\title{
Effect of Cortisol on Bovine Oocytes Maturation and Further Embryonic Development After In vitro Fertilization
}

\author{
Aya KH Mahdy², Maria Helena Moreira da Silva² and Fernando Moreira da Silva*1,2 \\ ${ }^{1}$ Faculty of Agrarian Sciences and Environment, Portugal \\ ${ }^{2}$ Department of Animal Reproduction, Portugal
}

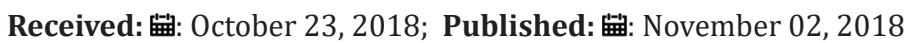

*Corresponding author: Fernando Moreira da Silva, Faculty of Agrarian Sciences and Environment, Department of Animal Reproduction, Portugal

\begin{abstract}
Oocyte meiotic maturation and further embryonic development after fertilization is one of the most important physiological requirements for species survival. Herein, the aim of the study was to evaluate the effects of the stressful hormone, cortisol, on the nuclear maturation and further embryonic development of bovine oocytes after in vitro fertilization (IVF). For such purpose, 1,439 immature oocytes were collected from slaughtered cows and matured in vitro for 24 hours with different concentrations of cortisol (0 (control); $50 \mu \mathrm{M} ; 150 \mu \mathrm{M} ; 250 \mu \mathrm{M}$ ). Afterwards, 412 oocytes were denuded, dyed with aceto-orcein and evaluated for meiotic development. The other 1027 were submitted to IVF and cultured for 9 days, being evaluated on day 2, 6 and 9, for cleavage, morula and blastocyst, respectively. In the control, $85 \%$ of oocytes reached Metaphase II, decreasing to 49,32 and $15 \%$ for the concentration of the cortisol $(50,150$, and $250 \mu \mathrm{M}$, respectively). For the embryos, obtained from the oocytes submitted to IVF, in the control group, $28.3 \pm 4.8 \%$ reached the stage of blastocyst, while for the concentrations of cortisol this value decreased to $22.1 \pm 5.4 \%, 15.4 \pm 6.0 \%$ and $6.5 \pm 2.1 \%$ for 50,150 and $250 \mu \mathrm{M}$ of cortisol, respectively). Results of the present study clearly demonstrated that animal's stress and particularly high concentrations of cortisol impair bovine nuclear maturation as well as the further embryonic development after IVF.
\end{abstract}

Keywords: Cortisol, In Vitro Oocyte Nuclear Maturation, IVF, Embryo Development.

Abbreviations: IVF: In Vitro Fertilization; GVBD: Germinal Vesicle Breakdown; MPF: Maturation Promoting Factor; ERKs: Extracellular SignalRegulated Kinases; GnRH: Gonadotropin-Releasing Hormone; FSH: Follicle-Stimulating Hormone; LH: Luteinizing Hormone; HPA: HypothalamicPituitary-Adrenal; DPBS: Dulbecco's Phosphate Buffered Saline; COCs: Cumulus Oocyte Complexes; FBS: Foetal Bovine Serum; GV: Germinal Vesicle; GVBD: Germinal Vesicle Break-Down; NCM: Non-Capacitating HEPES-Buffered Medium; TALP: Tyroide's Albumin Lactate Pyruvate; TNF: Tumor Necrosis Factor; FasL: Fas Ligand; NMR: Nuclear Maturation Rate; MAPK: Mitogen-Activated Protein Kinases.

\section{Introduction}

Stress is a process stimulus activate the entire system and produces an organic response generating negative effects on animal health and production. The hormone mainly produced during stress is the cortisol (C21H3005) which is secreted by the upper part of the adrenal gland, being an useful indicator as a biomarker to detect stress on the animals [1]. Besides, cortisol plays an important role during the catabolic phase and it negative effect on several metabolism has been well described [2], it is not yet clear the role of this hormone in ovaries and particularly on oocyte nuclear maturation and further embryo development after fertilization. The immature oocytes begin to develop in the ovaries, possessing a large nucleus referred to as germinal vesicle (GV), in which a sequence called germinal vesicle breakdown (GVBD), initiates the process of nuclear oocyte maturation, finishing at the stage of metaphase II just before ovulation [3]. Maturation promoting factor (MPF) is activated at GVBD and increases until it reaches a plateau at the end of the Metaphase I. A transient decline in MPF activity takes place during the transition between meiosis I, arresting at metaphase II.

During oocyte maturation, the extracellular signal-regulated kinases (ERKs) are activated and a comprehensive, extensive rearrangement of the cytoskeleton and associated proteins occurs involving a spindle pole close to the cortex [4-6]. After polar body extrusion, chromosomes realign progressing to metaphase II. All 
meiosis developmental stages occur when follicles are growing from preantral to antral follicles. Moreover, the ovulation occurs when oocyte is in the metaphase II stage [7-8]. At the endocrine level, folliculogenesis is regulated by a central nervous system, anterior pituitary, and ovary cascade mechanism. Specialized hypothalamic neurons secrete pulses of gonadotropin-releasing hormone (GnRH) into the portal blood vessels, which acts on the gonadotrophs to cause a pulsatile release of follicle-stimulating hormone (FSH) and luteinizing hormone ( $\mathrm{LH})$, which act on ovarian follicle cells to control folliculogenesis. Although GnRH, FSH, and LH are critically important in regulating folliculogenesis, hormones and growth factors, which are themselves products of the follicle, can act locally to modulate (amplify or attenuate) FSH and LH action [9-10].

This is the autocrine/paracrine system of developing follicles. It is believed that this local regulatory system plays an important role in the complex mechanisms governing the timing of folliculogenesis and whether a follicle becomes dominant or atretic. An important point is that estradiol produced by the dominant follicle rises production of GnRH, FSH and LH, increasing follicular growth, leading to its rupture/ovulation [11-13]. Stress-like levels of cortisol suppress follicular growth and development and block or delay the preovulatory surge of LH when cortisol is present during the late luteal and early follicular phases of the oestrous cycle [14-16]. In fact, since the last century it has been postulated that stressful stimuli reduce fertility in domestic species, such climatic extremes, transportation or laparoscopy as well as psychological stress suppress or delay expression of behavioural oestrus and ovulation. In addition to reducing fertility, these stressors also stimulate the activity of the hypothalamic-pituitary-adrenal (HPA) axis, and a marked increase in serum concentration of cortisol is commonly associated with management-related stressors [17-19].

Furthermore, cortisol reduces amplitude of GnRH and LH secretion and lowers plasma estradiol levels in follicular-phase and for such reason, high cortisol levels can inhibit the reproduction physiology [20-21]. The effect of cortisol inhibition in the protein ERKs disrupting their functions in meiotic maturation of fullgrown oocytes and/or arrest at metaphase of meiosis II prior to fertilization [22-23]. Although the causal link between stress and infertility has not been precisely defined, several studies indicate that glucocorticoids in general and cortisol in particular may contribute to the anti-gonadal effect of stress [24-25]. Additionally, the increasing of cortisol level reduces estradiol production possibly by affecting the granulosa cell functions within the follicle, which results deterioration in oocyte quality, leading to a poorest ability to develop after fertilization [17]. The aim of the present study is to evaluate the role of this hormone on bovine in vitro oocyte nuclear maturation and further embryo development after fertilization.

\section{Materials and Methods}

\section{Chemicals}

All the chemicals and reagents used in this study were obtained from Sigma-Aldrich (St. Louis, Mo, USA) unless stated otherwise.

\section{Ovaries}

Ovaries ( $\mathrm{n}=350)$ were obtained at a local abattoir from adult animals, trimmed of adhering tissue and transported to the laboratory in Dulbecco's phosphate buffered saline (DPBS), at temperature ranging from 35 to $37^{\circ} \mathrm{C}$ within 2 hours postslaughtering.

\section{Experimental Design}

\section{In Vitro Oocyte Nuclear Maturation}

Cumulus oocyte complexes (COCs) were washed twice in TCM199 medium supplemented with 2\% Foetal Bovine Serum (FBS), $0.3 \mathrm{mg} / \mathrm{ml}$ glutamine and $50 \mathrm{mg} / \mathrm{ml}$ gentamicin and then washed twice in maturation medium supplemented with 10\% FBS, $5 \mu \mathrm{g} /$ $\mathrm{ml}$ of FSH-LH, $1 \mu \mathrm{g} / \mathrm{ml}$ estradiol-17ß, $0.15 \mathrm{mg} / \mathrm{ml}$ glutamine, $22 \mu \mathrm{g} /$ $\mathrm{ml}$ Na-pyruvate, $50 \mu \mathrm{g} / \mathrm{ml}$ gentamicin. Then, COCs were transferred to sterile Petri dishes containing $100 \mu \mathrm{l}$ of maturation medium supplemented with cortisol, previously diluted in ethanol at 0 (control) 50, 150 and $250 \mu \mathrm{M}$ final concentration (10-15 oocytes/ droplet) at in an incubator (Lab line instrument Inc. USA) at 38.5 ${ }^{\circ} \mathrm{C}$ for 22-24 hours with saturated humidity and $5 \%$ of $\mathrm{CO}_{2}$, for maturation. Afterwards, COCs were selected, and divided into two groups: ( 1 and 2). The ones considered in the group A $(n=412)$ were fixated and stained for meiosis development evaluation, while the ones considered in the group B $(n=1027)$ were used for IVF.

\section{Oocyte Fixation and Nuclear Staining}

For meiosis development evaluation, COCs were selected, denuded by vortexing for 2 min and fixated in 3:1 (Methanol: glacial acetic acid) solution for $24 \mathrm{~h}$ at room temperature in petri dishes sealed with parafilm and then dyed with $1 \%$ Orcein. Meiosis stages were recorded as previously described [26] under a phase contrast microscope at 400x magnification. Meiotic stages were classified as germinal vesicle (GV), germinal vesicle break-down (GVBD), metaphase I (MI), anaphase I (Al), telophase I (TI), and metaphase II (MII) [27], being considered as mature those evaluated on Telophase I to Metaphase II.

\section{IVF}

After 24 hours of maturation, oocytes designated to IVF were selected under the stereomicroscope, transferred into a Petri dish containing maturation medium and washed twice in IVF medium. After thawing, semen was washed twice by centrifuge at $320 \mathrm{xg}$ for 5 minutes each, in non-capacitating HEPES-buffered medium (NCM) sperm washing medium (5 $\mathrm{ml}$ each time). After removing the supernatant, sperm pellet was homogenized by pipetting in the remaining NCM Sperm wash medium (0.25-0.5 ml) for adjusting the sperm concentration and motility. A volume of $20 \mu$ of the semen (11.5 x 106) was uploaded on a slide, covered with a cover slip and the motility of the sperms was checked under the inverted microscope. To adjust the semen concentration, $5 \mu \mathrm{l}$ from the semen were added to $95 \mu \mathrm{l}$ distilled water in an Eppendorf tube, and then exposed to direct light until the semen died. Oocytes and sperms were then co-cultured in $500 \mu \mathrm{l}$ of IVF Tyroide's Albumin Lactate 
Pyruvate (TALP) for $22-24$ hours at $38.5^{\circ} \mathrm{C}$ in the incubator with saturated humidity and $5 \%$ of $\mathrm{CO} 2$

\section{In Vitro Embryo-Culture (IVC)}

Twenty-four hours from the fertilization, presumptive zygotes were transferred to a glass tube containing $1 \mathrm{ml}$ of washing medium and vortexed to remove granulosa cells attached them. Then the embryos were checked under the microscope, washed twice in culture medium and cultured for 9 days.

\section{Statistical Analysis}

Data are expressed as percentages, and they were subjected to arcsine transformation when required by $\mathrm{X}^{\prime}=\arcsin \sqrt{\mathrm{x}}$ All the statistical analyses were performed by one-way ANOVA in SPSS vs 22. When significant differences were found, comparison between treatments was made using either the post hoc, to verify differences between the groups, followed by the LSD test between groups when parametric assumptions were not fulfilled. Results are expressed as mean \pm SEM. Probability values less than 0.05 were considered statistically different.

\section{Results and Discussion}

]In the present study the effect of several concentrations of cortisol, ranging from 0 to $250 \mu \mathrm{M}$ was evaluated on the nuclear and further in vitro development of bovine oocytes after IVF. As known, cortisol is a glucocorticoid hormone, which plays an important role in numerous processes including metabolism, blood pressure, and immune response regulation, and thus has proved a reliable biological correlate of many adverse health outcomes [33]. In that manner, as cortisol is considered a stressful hormone an effective negatively for the maturation oocytes would be expected on oocytes maturation and further embryonic development after IVF [34]. In fact, several studies have observed exposing oocytes to high concentration of cortisol decreased their competence, undergo impair oocyte developmental potential by triggering apoptosis of ovarian cells via activating a transmembrane protein belonging to the tumor necrosis factor (TNF) usually known as Fas ligand (FasL). Research developed on mice were injected with cortisol, indicated a clear negative impact of cortisol on oocyte's nuclear maturation rate (NMR), blastocyst rates and the cell number per blastocyst [3536].

Furthermore, related for pigs that significant high concentration of cortisol in unfertilized oocytes leads to its relative decrease in the NMR, fertilization or embryo cleavage [37]. Several research developed in vivo show cortisol as a stressful hormone, affects bovine metabolism, in general, and reproduction in particular [30-31]. Previously studies showed that in the Azores, fertility of bovine decreases drastically to $35 \%$ in the summer period, achieving $78 \%$ in the non-hot periods as spring and autumn [4445]. Several hypotheses have been purposed to explain this shut in the hot periods [18, 32, 45]. Heat stress genes, such HASP14 and Cx34 are activated during summer, when outside temperature rises over $25 \stackrel{\circ}{\circ}$. As these genes are also related to the apoptosis, one can speculate that, among other mechanisms, apoptosis can play a crucial role in the embryonic as well as in the follicle/oocyte development from the beginning to metaphase II [32, 45]. Besides in the literature, cortisol levels on cattle are not very clear, these values were chosen according several authors in which cortisol can reach a maximum of about $250 \mu \mathrm{M}$ when cattle are exposed for long time to stress conditions such high environmental temperature [28-29].

From the 1439 oocytes used in this experiment, results revealed that those $(n=412)$ employed for maturation rate, a statistical decreased was observed when cortisol levels increased in the maturation medium. For cortisol levels of 0 (control); $50 \mu \mathrm{M} ; 150$ $\mu \mathrm{M} ; 250 \mu \mathrm{M}$ groups, rate of maturation was respectively 85.0; 49.0; 32.0 and 15.0 (Table 1). A negative correlation $(\mathrm{R} 2=0.991)$ was observed between these two parameters $(\mathrm{P}<0.01)$ (Figure 1). For the 1027 oocytes used for IVF, a statistical $(\mathrm{p}<0.01)$ negative doseresponse was observed between the number of produced embryos in the stage of blastocysts and the cortisol concentrations (Table 2).

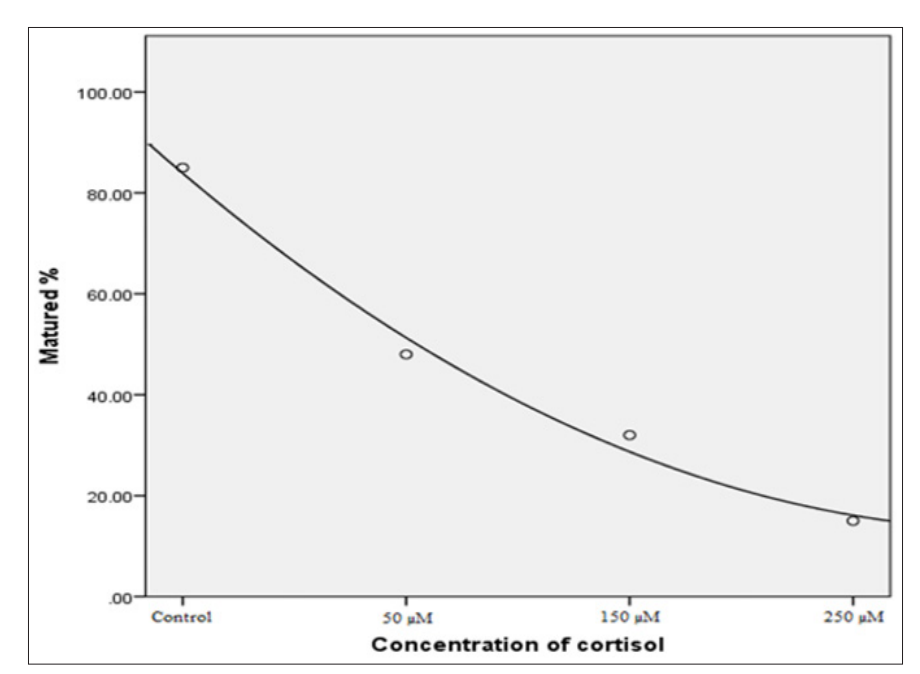

Figure 1: Correlation between mature oocytes and the different concentrations of cortisol. $\left(Y=1.02+-47.6^{*} X+5^{*} X 2, R 2=0.991\right.$ $\mathrm{p}<0.01)$. 
Table 1: Nuclear development stages, after maturation with cortisol.

\begin{tabular}{|c|c|c|c|c|c|c|}
\hline \multirow{3}{*}{ Cortisol Concentrations $(\mu \mathrm{M})$} & \multirow{3}{*}{ Number of oocytes } & \multicolumn{5}{|c|}{ Meiosis Development (\%) } \\
\hline & & \multirow{2}{*}{ GV } & \multirow{2}{*}{ GVBD } & \multirow{2}{*}{ MI } & \multirow{2}{*}{ AI } & Nuclear maturation rate \\
\hline & & & & & & (TI + MII) \\
\hline 0 (Control) & 138 & 1.4 & 2.2 & 12.0 & 0 & 85.0 \\
\hline 50 & 91 & 2.2 & 4.4 & 18.0 & 26.0 & 49.0 \\
\hline 150 & 97 & 2.1 & 5.2 & 53.0 & 8.0 & 32.0 \\
\hline 250 & 86 & 2.3 & 11.6 & 57.0 & 14.0 & 15.0 \\
\hline
\end{tabular}

Note: GV-Germinal Vesicle; AI- Anaphase I GVBD- Germinal Vesicle Break Down; TI- Telophase MI; Metaphase I; MII- Metaphase II

Table 2: Embryonic developmental stages of oocytes $(n=1027)$ submitted to different cortisol concentrations during maturation. Results the mean \pm SEM) of six replicas. Different letters $(a, b$, and $c)$, indicate statistical differences $(p<0.01)$. Day 0 is the fertilization day.

\begin{tabular}{|c|c|c|c|c|}
\hline Treatment & Number of oocytes & Cleavage (\%) (Day 2) & Morula(\%) (Day 6) & Blastocyst (\%) (Day 9) \\
\hline Control & 180 & $72.2 \pm 3.9^{\mathrm{a}}$ & $41.1 \pm 5.4^{\mathrm{a}}$ & $28.3 \pm 4.8^{\mathrm{a}}$ \\
\hline $50 \mu \mathrm{M}$ & 263 & $56.3 \pm 1.2^{\mathrm{a}}$ & $31.6 \pm 5.1^{\mathrm{a}}$ & $22.1 \pm 5.4^{\mathrm{a}}$ \\
\hline $150 \mu \mathrm{M}$ & 292 & $47.6 \pm 5.2^{\mathrm{a}}$ & $26.0 \pm 8.0^{\mathrm{a}}$ & $15.4 \pm 6.0^{\mathrm{b}}$ \\
\hline $250 \mu \mathrm{M}$ & 292 & $27.4 \pm 2.7^{\mathrm{c}}$ & $11.3 \pm 1.1^{\mathrm{c}}$ & $6.5 \pm 2.1^{\mathrm{c}}$ \\
\hline
\end{tabular}

In our study, cortisol significantly affected NMR decreasing to $15.0 \%$ and blastocyst production to $6.5 \%$ when oocytes were exposed to $250 \mu \mathrm{M}$ of cortisol $(\mathrm{P}<0.01)$, which values are in agreement with those published also working with cattle [38]. Moreover, in an endeavour to explain the clear inhibitory effect of cortisol, Gonzalez and collaborators [2] explained that corticosterone decreases phosphorylated forms of extracellular signal-regulated kinases (ERKs), phospho-ERK (p-ERK)-1 and p-ERK-2 in exposed oocytes and this could represent an alteration in molecular mechanisms underlying oocyte maturation and competence for subsequent development.

The ERKs are members of the mitogen-activated protein kinases (MAPK) family activated during oocyte maturation. The MAPKs together with maturation/metaphase-promoting factor (MPF complex), the two important protein kinases for oocyte meiosis, interact intimately and perform essential regulating roles in meiotic maturation and fertilization [39]. Additionally, in mammalian oocytes, several kinases, such as Plk1- and Akt-induced, regulates the MPF activation, which plays a key role in the events of GVBD. Moreover, PKA can activate Wee1B (Wee1-like protein kinase 1B) or inhibit CDC25B, which downregulates MPF activity and prevents GVBD [40-41]. This mechanism is independent of MAPK activation inside oocytes [42]. In porcine oocytes, the inhibitory action of cortisol on meiotic maturation could be due to the reduction in p34cdc2-cyclin B1 complex, one of the components of MPF [43]. In addition, results presented and demonstrated that the effect of high ambient temperatures on reproductive processes, decrease the bovine fertility to $36.8 \%$ in the summer period, affecting also the physiological mechanisms, inhibiting the in vitro oocytes maturation rate to $44.3 \%$ while the in vitro embryonic development in that period was $19.6 \%$ [44].

In another research developed by our working group, these authors, showed that heat stress influences the expression on some genes related to growth and develop embryos such $\mathrm{Cx} 43, \mathrm{CDH} 1$, DNMT1, HSPA14, in which the lower maturation rate could may be due to the down-regulation of these genes. Whereas, HSPA14 gene is an important part of the cell's machinery for folding, unfolding, transport, localization of proteins and differentiation, regulation of the embryonic cell cycle and helping to protect cells from stress. Therefore, HSPA14 is an apoptotic gene induced by heat shock is associated with embryonic loss, playing an important role of control mechanism of processes involved in growth, cellular differentiation, and embryonic development [45]. Furthermore, $\mathrm{Cx} 43$ is expressed in numerous tissues including gonads, act as a mediator of heat stress effect on cells [18]. Additionally, plasma concentrations of cortisol significantly increased following exposure to heat stress $[18,32]$. Under conditions of prolonged heat exposed to a moderate heat stress $\left(35^{\circ} \mathrm{C}\right)$ cow's plasma cortisol increased significantly $(\mathrm{P}<0.05)$.

Therefore, plasma cortisol may increase within $20 \mathrm{~min}$ of exposure to acute heat stress and reach a plateau within $2 \mathrm{~h} \mathrm{[46].}$ Previously research demonstrated clear the viewpoint of animal welfare in both of the high ambient temperatures, high direct and indirect solar radiation, showing an unequivocal evidence that hyperthermia is deleterious to any form of productivity [47]. Further, numerous studies that use behavioural indicators of welfare show that behavioural changes can be interpreted as either good or poor welfare and can be defined by discrete measures, such as changes in hormone level, body temperature, and normal behaviour [48]. The type of biological defence an animal utilizes is not important but the resulting change in biological function determines if there is a threat to the animal welfare [49].

\section{Conclusion}

Results of the present research clearly demonstrated that animal's stress and particularly cortisol levels might have impaired 
ovulation and oocyte potential, influencing negatively their ability to develop to the stage of blastocyst after fertilisation. Management practices such as handling, weaning, housing conditions and transportation are essential to reproduction efficiency of cattle.

\section{Acknowledgment}

This project was financed in $85 \%$ by FEDER and in $15 \%$ with regional funds through the Programa Operacional Açores 2020 (Operational Program Azores 2020), in scope of the project «BEMAP-ET - ACORES-01-0145-FEDER-000026».

\section{References}

1. Martínez Miró S, Tecles F, Ramón M, Escribano D, Hernández F, et al. (2016) Causes, consequences and biomarkers of stress in swine: an update. BMC Vet Res 12:171.

2. González R, Ruiz León Y, Gomendio M, Roldan R (2010a) The effect of glucocorticoids on mouse oocyte in vitro maturation and subsequent fertilization and embryo development. Toxicology In Vitro 24: 108-115.

3. Chen Z, Ming T, Nielsen H (2010) Maturation arrest of human oocytes at germinal vesicle stage. J Hum Reprod Sci 3(3): 153-157.

4. Brunet S, Maro K (2005) Cytoskeleton and cell cycle control during meiotic maturation of the mouse oocyte: integrating time and space. Reproduction 130(6): 801-811.

5. Kwon S, Lim J (2011) Small GTPases and formins in mammalian oocyte maturation: cyto- skeletal organizers. Clin Exp Reprod Med 38(1): 1-5.

6. Sojung K, Hyunjung J (2011) Small GTPases and formins in mammalian oocyte maturation: cytoskeletal organizers. Clin Exp Reprod Med 38(1): $1-5$.

7. Palma G, Argañaraz M, Barrera A Rodler D, Mutto A, Sinowatz F, et al. (2012) Biology and Biotechnology of Follicle Development. Scientific World Journal 2012: 938138.

8. Christensen A, Bentley G, Cabrera R, Ortega H, Perfito N, et al. (2012) Hormonal Regulation of Female Reproduction. Horm Metab Res 44(8): 587-591.

9. Kumar P, Sharma A (2014) Gonadotropin-releasing hormone analogs: Understanding advantages and limitations. J Hum Reprod Sci 7(3): 170174.

10. Hsueh J, Mc Gee A, Hayashi M, Hsu Y (2000) Hormonal regulation of early follicle development in the rat ovary. Mol Cell Endocrinol 163(12): 95-100.

11. Gittens J, Barr K, Vanderhyden B, Kidder G (2005) Gap junctions and paracrine signaling in the ovary. Journal of Cell Science. 118(1): 113-122.

12. Mc Gee A, Raj S (2015) Regulators of ovarian preantral follicle development. Semin Reprod Med 33(3): 179-184.

13. Macfarlane S Breen M Sakurai H, Adams M, Adams E (2000) Effect of duration of infusion of stress-like concentrations of cortisol on follicular development and the preovulatory surge of LH in sheep. Anim Reprod Sci 63(3-4): 167-75.

14. Welsh R, Johnson H (1981) Stress-induced alterations in secretion of corticosteroids, progesterone, luteinizing hormone, and testosterone in bulls. Endocrinology 109(1): 185-190.

15. Wilson J, Marion S, Spain N, Spiers E, Keisler H, et al. (1998) Effects of controlled heat stress on ovarian function of dairy cattle. 1. Lactating cows. J Dairy Sci 81(8): 2124-2131.

16. Doney M, Gunn G, Griffiths G (1973) The effect of premating stress on the onset of oestrus and on ovulation rate in Scottish blackface ewes. J Reprod Fertil 35(2): 381-384.
17. Prasad S, Tiwari M, Pandey A, Shrivastav T, Chaube S (2016) Impact of stress on oocyte quality and reproductive outcome. J Biomed Sci 23:36.

18. Mahdy A, Pavani K, Baron E, Da Silva F (2017) Studies on Gene Expression and Developmental Competence of Bovine Embryos Produced Under Different Conditions of Heat Stress: Full Chapter. Trends and Advances in Veterinary Genetics. In Tech Open 3: 30-60.

19. Komesaroffet A, Esler M, Clarke J, Fullerton J, Funder W (1998) Effects of estrogen and estrous cycle on glucocorticoid and catecholamine responses to stress in sheep. Am J Physiol 275: E671-E678.

20. Wagenmaker E, Breen K, Oakley A, Pierce B, Tilbrook A, et al. (2009) Cortisol Interferes with the Estradiol-Induced Surge of Luteinizing Hormone in the Ewe. Biol Reprod 80(3): 458-463.

21. De Graaf Roelfsema E, Keizer A, Van E, Wijnberg D, Van H (2007) Hormonal responses to acute exercise, training and overtraining. A review with emphasis on the horse. Vet Q 29(3): 82-101.

22. Lotfi C, Mendonca P (2016) Comparative Effect of ACTH and Related Peptides on Proliferation and Growth of Rat Adrenal Gland. Front Endocrinol (Lausanne) 7: 39.

23. Lee M, Ohmachi M, Arur S, Nayak S, Francis R, et al. (2007) Multiple Functions and Dynamic Activation of MPK-1 Extracellular SignalRegulated Kinase Signaling in Caenorhabditis elegans Germline Development. Genetics 177(4): 2039-2062.

24. Dobson H, Smith F (1995) Stress and reproduction in farm animals. J Reprod Fertil 49: 451-461.

25. Chrousos P, Torpy J, Gold W (1998) Interactions between the hypothalamic-pituitary-adrenal axis and the female reproductive system: clinical implications. Ann Intern Med 129(3): 229-240.

26. Faheem MS, Carvalhais A Chaveiro I, Moreira da Silva F (2011) In vitro oocyte fertilization and subsequent embryonic development after cryopreservation of bovine tissue, using an effective approach for oocyte collection. Anim Reprod Sci 125: 49-55.

27. Amini E, Asadpour R, Roshangar L, Jafari Joozani R (2016) Effect of linoleic acid supplementation on in vitro maturation, embryo development and apoptotic related gene expression in ovine. Int J Reprod Biomed (Yazd) 14(4): 255-262.

28. Polsky L, Von Keyserlingk M (2017) Invited review: Effects of heat stress on dairy cattle welfare. J Dairy Sci 100(11): 8645-8657.

29. González R, Ruiz León Y, Gomendio M, Roldan R (2010b) The effect of glucocorticoids on ERK-1/2 phosphorylation during maturation of lamb oocytes and their subsequent fertilization and cleavage ability in vitro. Reprod Toxicol 29(2): 198-205.

30. Ojima M, Satoh K, Gomibuchi T, Itoh N, Kin S, et al. (1990) The inhibitory effects of glycyrrhizin and glycyrrhetinic acid on the metabolism of cortisol and prednisolone--in vivo and in vitro studies. Nihon Naibunpi Gakkai Zasshi 66(5): 584-596.

31. Mudroň P, Rehage J, Sallmann H, Höltershinken M, Scholz H (2005) Stress Response in Dairy Cows Related to Blood Glucose. Acta Vet Brno 74(1): 37-42.

32. Pavani K, Baron E, Correia P, Lourenço J, Bettencourt B et al. (2016) Gene expression, oocyte nuclear maturation and developmental competence of bovine oocytes and embryos produced after in vivo and in vitro heat shock Zygote 24(5): 748-759.

33. Massey J, Campbell B, Raine Fenning N, Aujla K, Vedhara K (2014) The association of physiological cortisol and IVF treatment outcomes: a systematic. Reprod Med Biol 13(4): 161-176.

34. Ranabir S, K Reetu (2011) Stress and hormones. Indian J Endocrinol Metab 15(1): 18-22.

35. Raghavendra B, Sreenivasan Y, Manna K (2007) Oleandrin induces apoptosis in human, but not in murine cells: dephosphorylation of Akt, 
expression of FasL, and alteration of membrane fluidity. Mol Immunol 44(9): 2292-2302.

36. Yuan H, Han X, He N, Wang G, Gong S, et al. (2016) Glucocorticoids impair oocyte developmental potential by triggering apoptosis of ovarian cells via activating the Fas system. Sci Rep 6: 24036.

37. Andersen C (2003) Effect of glucocorticoids on spontaneous and folliclestimulating hormone induced oocyte maturation in mouse oocytes during culture. J Steroid Biochem Mol Biol 85(2-5): 423-427.

38. Macedo G, Zúccari C, De Abreu U, Negrão J, Da Costa e Silva E (2011) Human-animal interaction, stress, and embryo production in Bos indicus embryo donors under tropical conditions. Trop Anim Health Prod 43(6): 1175-1182.

39. Fan H, Sun Q (2004) Involvement of mitogen-activated protein kinase cascade during oocyte maturation and fertilization in mammals. Biol Reprod 70: 535-547.

40. Han J, Chen R, Paronetto P, Conti M (2005) Wee1B is an oocyte-specific kinase involved in the control of meiotic arrest in the mouse. Curr Biol 15(18): 1670-1676.

41. Han J, Conti M (2006) New pathways from PKA to the Cdc2/cyclin B complex in oocytes: Wee1B as a potential PKA substrate. Cell Cycle 5(3): 227-231.

42. Liang C, Su Y, Fan H, Schatten H, Sun Q (2007) Mechanisms regulating oocytes meiotic resumption: roles of mitogen-activated protein kinase. Molecular Endocrinology 21(9): 2037-2055.

\section{ISSN: 2574-1241}

DOI: 10.26717/BJSTR.2018.10.001998

Fernando Moreira da Silva. Biomed J Sci \& Tech Res

(c) (i) This work is licensed under Creative

Submission Link: https://biomedres.us/submit-manuscript.php
43. Chen W, Yang J, Li P (2000) Effect of dexamethasone on the expression of p34cdc2 and cyclin B1 in pig oocytes in vitro. Molecular Reproduction Development 56(1): 74-79.

44. Pavani K, Carvalhais I, Faheem M, Chaveiro A, Reis V, et al. (2015) Reproductive Performance of Holstein Dairy Cows Grazing in Drysummer Subtropical Climatic Conditions: Effect of Heat Stress and Heat Shock on Meiotic Competence and In vitro Fertilization. Asian-Australas J Anim Sci 28(3): 334-342.

45. Pavani K, Rocha A, Baron E, Lourenço J, Faheem M, et al. (2017) The effect of kinetic heat shock on bovine oocyte maturation and subsequent gene expression of targeted genes. Zygote 25(3): 383-389.

46. Christison G, Johnson H (1972) Cortisol turnover in heat-stressed cows. Journal of Animal Science Abstract 35(5): 1005-1010.

47. Silanikove N (2000) Effects of heat stress on the welfare of extensively managed domestic ruminants. Livestock Production Science 67(1-2): $1-18$.

48. Martins C, Galhardo L, Noble C, Damsgård B, Spedicato M, et al. (2012). Behavioural indicators of welfare in farmed fish. Fish Physiol Biochem 38(1): 17-41.

49. Pampori Z, Huozha R, Shah K, Andrabi S, Amin T (2010) Stress Versus Reproduction in Animals. Vetscan 5(2): 61.

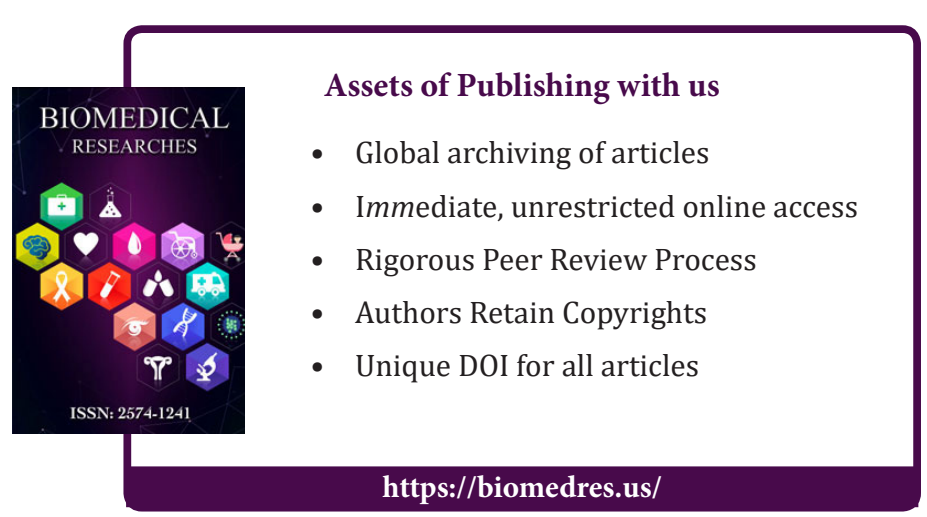

